Vietnam Journal of Mechanics, VAST, Vol.40, No. 1 (2018), pp. 63 - 78

DOI:10.15625/0866-7136/10417

\title{
H/V RATIO OF RAYLEIGH WAVES IN COMPRESSIBLE PRE-STRESSED ELASTIC HALF-SPACES COATED WITH A THIN LAYER: AN APPROXIMATE FORMULA
}

\author{
Nguyen Thi Khanh Linh ${ }^{1}$, Pham Chi Vinh ${ }^{2, *}$, Le Thi Hue ${ }^{3}$ \\ ${ }^{1}$ Thuyloi University, Hanoi, Vietnam \\ ${ }^{2}$ VNU University of Science, Hanoi, Vietnam \\ ${ }^{3}$ Vietnam National University of Forestry, Hanoi, Vietnam \\ *E-mail: pcvinh@vnu.edu.vn \\ Received July 01, 2017
}

\begin{abstract}
This paper is concerned with the propagation of Rayleigh waves in a compressible pre-stressed elastic half-space coated with a thin compressible pre-stressed elastic layer. The main purpose of the paper is to establish an approximate formula for the $\mathrm{H} / \mathrm{V}$ ratio (the ratio between the amplitudes of the horizontal and vertical displacements of Rayleigh waves at the traction-free surface of the layer). First, the relations between the traction amplitude vector and the displacement amplitude one of Rayleigh waves at two sides of the interface between the layer and the half-space are created. From the continuity condition at the interface and these relations the displacement amplitude vector of Rayleigh waves at the interface is determined. Then, a third-order approximate formula for the $\mathrm{H} / \mathrm{V}$ ratio has been derived by expanding the layer displacement amplitude vector at its traction-free surface into Taylor series at the interface. It is shown numerically that the obtained formula is a good approximate one.
\end{abstract}

Keywords: Rayleigh waves, the H/V ratio, compressible, pre-stressed, approximate formula for the $\mathrm{H} / \mathrm{V}$ ratio.

\section{INTRODUCTION}

As addressed by Junge et al. [1], the H/V ratio (the ratio between the amplitudes of the horizontal and vertical displacements of Rayleigh waves at the traction-free surface of half-spaces) is more sensitive than the Rayleigh wave velocity as an indicator of the state of stress, and, in contrast with the Rayleigh wave velocity, it is reference free. Therefore, the $\mathrm{H} / \mathrm{V}$ ratio of Rayleigh waves is a convenient tool for nondestructively evaluating pre-stress of structures before and during loading. For extracting the pre-stress from the experimentally measured values of the $\mathrm{H} / \mathrm{V}$ ratio (the inverse problem) we need an

(C) 2018 Vietnam Academy of Science and Technology 
explicit formula for the $\mathrm{H} / \mathrm{V}$ ratio which is the mathematical base for solving the inverse problem.

While a large number of explicit formulas for the Rayleigh wave velocity have been derived, see for examples, [2-14], a few of formulas for the H/V ratio have been obtained. These are the $\mathrm{H} / \mathrm{V}$ ratio formulas for an isotropic compressible half-space with traction-free surface [15], for an isotropic compressible half-space subject to an impedance boundary condition [15], for an isotropic compressible half-space coated by an isotropic compressible half-space layer [15] and for an isotropic incompressible half-space coated by an isotropic incompressible half-space layer [16].

In this paper, an approximate formula for the $\mathrm{H} / \mathrm{V}$ ratio of third-order in terms of dimensionless thickness of the layer has been established. This formula is derived by using the relations between the traction and displacement amplitude vectors of Rayleigh waves at two sides of the welded interface between the layer and the half-space, along with the Taylor expansion of the displacement amplitude vector of the thin layer at its traction-free surface. It is shown numerically that the obtained approximate formula is a good approximation. As the structures of a thin film attached to solids, modeled as half-spaces coated with a thin layer, are widely used in the modern technology, the obtained approximate formula is significant in nondestructively evaluating pre-stress of structures.

It is also note that the $\mathrm{H} / \mathrm{V}$ ratio is an important parameter which reflects fundamental properties of the elastic material [15], it can be thus used for the nondestructive evaluation of the elastic constants of material [17], beside its well-known applications in seismology $[18,19]$.

\section{RELATIONS BETWEEN THE TRACTION AND DISPLACEMENT AMPLITUDE VECTORS AT TWO SIDES OF THE INTERFACE}

\subsection{Basic equations for a compressible pre-stressed elastic layer in matrix form}

In the deformed configuration, we consider a compressible pre-stressed elastic layer $-h \leq x_{2} \leq 0$ of uniform thickness $h$ overlying a compressible pre-stressed half-space $x_{2} \geq 0$. Both the layer and the half-space are subject to a pure homogeneous pre-strain (see, Dowaikh \& Ogden [3] and Ogden [4]) and the principal directions of pre-strain of the layer and half-space are aligned, one direction being normal to the planar interface $x_{2}=0$. Note that a rectangular Cartesian coordinate system $x_{1}, x_{2}, x_{3}$ is employed with its axes coinciding with the principal directions of the pre-strain. The principal stretches of the initial deformation of the half-space and the layer are denoted by $\lambda_{1}, \lambda_{2}, \lambda_{3}$ and $\bar{\lambda}_{1}, \bar{\lambda}_{2}, \bar{\lambda}_{3}$, respectively. They are positive constants. The layer is assumed to be perfectly bonded to the half-space. Note that quantities related to the half-space and the layer have the same symbol but are systematically distinguished by a bar if pertaining to the layer. An incremental (infinitesimal) motion in the $x_{1}, x_{2}$-plane is now superimposed on the underlying initial deformations, with its displacement components in the half-space and the layer being independent of $x_{3}$ and denoted by $u_{1}, u_{2}$ and $\bar{u}_{1}, \bar{u}_{2}$, respectively. 
H/V ratio of Rayleigh waves in compressible pre-stressed elastic half-spaces coated with a thin layer: An approximate formula 65

For the layer, in the absence of body forces the equations of motion governing infinitesimal motion are $[3,4]$

$$
\begin{aligned}
& \bar{s}_{11,1}+\bar{s}_{21,2}=\bar{\rho} \ddot{\bar{u}}_{1}, \\
& \bar{s}_{12,1}+\bar{s}_{22,2}=\bar{\rho} \ddot{\bar{u}}_{2},
\end{aligned}
$$

where $\bar{\rho}$ is the mass density of material at the static deformed state, a superposed dot signifies differentiation with respect to $t$, commas indicate differentiation with respect to spatial variables $x_{k}$, and

$$
\bar{s}_{j i}=\bar{A}_{j i l k} \bar{u}_{k, l}
$$

$\bar{A}_{i j k l}$ are components of the fourth-order elasticity tensor defined as follows $[3,4]$

$$
\begin{gathered}
\bar{J} \bar{A}_{i i j j}=\bar{\lambda}_{i} \bar{\lambda}_{j} \frac{\partial^{2} \bar{W}}{\partial \bar{\lambda}_{i} \partial \bar{\lambda}_{j}}, \\
\bar{J} \bar{A}_{i j i j}=\left\{\begin{array}{l}
\left(\bar{\lambda}_{i} \frac{\partial \bar{W}}{\partial \bar{\lambda}_{i}}-\bar{\lambda}_{j} \frac{\partial \bar{W}}{\partial \bar{\lambda}_{j}}\right) \frac{\bar{\lambda}_{i}^{2}}{\bar{\lambda}_{i}^{2}-\bar{\lambda}_{j}^{2}}, \quad\left(i \neq j, \lambda_{i} \neq \lambda_{j}\right) \\
\frac{1}{2}\left(\bar{J} \bar{A}_{i i i i}-\bar{J} \bar{A}_{i i j j}+\bar{\lambda}_{i} \frac{\partial \bar{W}_{i}}{\partial \bar{\lambda}_{i}}\right) \quad\left(i \neq j, \bar{\lambda}_{i}=\bar{\lambda}_{j}\right) \\
\bar{J} \bar{A}_{i j j i}=\bar{J} \bar{A}_{j i i j}=\bar{J} \bar{A}_{i j i j}-\bar{\lambda}_{i} \frac{\partial \bar{W}}{\partial \bar{\lambda}_{i}}(i \neq j),
\end{array}\right.
\end{gathered}
$$

with $i, j \in\{1,2,3\}, \bar{W}=\bar{W}\left(\bar{\lambda}_{1}, \bar{\lambda}_{2}, \bar{\lambda}_{3}\right)$ is the strain-energy function per unit volume in unstressed state, $\bar{J}=\bar{\lambda}_{1} \bar{\lambda}_{2} \bar{\lambda}_{3}$.

In the stress-free configuration (3)-(5) reduce to

$$
\bar{A}_{i i i i}=\bar{\lambda}+2 \bar{\mu}, \quad \bar{A}_{i i j j}=\bar{\lambda}, \quad \bar{A}_{i j i j}=\bar{A}_{i j j i}=\bar{\mu},
$$

where $\bar{\lambda}, \bar{\mu}$ are the Lame moduli. For simplicity, we use the notations

$$
\begin{aligned}
& \bar{\alpha}_{11}=\bar{A}_{1111}, \bar{\alpha}_{22}=\bar{A}_{2222}, \bar{\alpha}_{12}=\bar{\alpha}_{21}=\bar{A}_{1122}, \\
& \bar{\gamma}_{1}=\bar{A}_{1212}, \bar{\gamma}_{2}=\bar{A}_{2121}, \bar{\gamma}_{*}=\bar{A}_{2112},
\end{aligned}
$$

(which are different from those defined by [3] by a factor $J$ ). In terms of these notations Eq. (2) becomes

$$
\begin{aligned}
& \bar{s}_{11}=\bar{\alpha}_{11} \bar{u}_{1,1}+\bar{\alpha}_{12} \bar{u}_{2,2}, \\
& \bar{s}_{22}=\bar{\alpha}_{12} \bar{u}_{1,1}+\bar{\alpha}_{22} \bar{u}_{2,2}, \\
& \bar{s}_{12}=\bar{\gamma}_{1}\left(\bar{u}_{2,1}+\bar{\gamma}_{*} \bar{u}_{1,2}\right), \\
& \bar{s}_{21}=\bar{\gamma}_{*}\left(\bar{u}_{2,1}+\bar{\gamma}_{2} \bar{u}_{1,2}\right) .
\end{aligned}
$$

From the strong-ellipticity condition, $\bar{\alpha}_{i k}$ and $\bar{\gamma}_{k}$ are required to satisfy the inequalities $[4,13]$

$$
\bar{\alpha}_{11}>0, \quad \bar{\alpha}_{22}>0, \quad \bar{\gamma}_{1}>0, \quad \bar{\gamma}_{2}>0 .
$$

Substituting (8) into (1) leads

$$
\begin{aligned}
& \bar{\alpha}_{11} \bar{u}_{1,11}+\bar{\gamma}_{2} \bar{u}_{1,22}+\left(\bar{\alpha}_{12}+\bar{\gamma}_{\star}\right) \bar{u}_{2,12}=\bar{\rho} \ddot{\ddot{u}}_{1}, \\
& \left(\bar{\alpha}_{12}+\bar{\gamma}_{\star}\right) \bar{u}_{1,12}+\bar{\gamma}_{1} \bar{u}_{2,11}+\bar{\alpha}_{22} \bar{u}_{2,22}=\bar{\rho} \ddot{\bar{u}}_{2} .
\end{aligned}
$$


From (8) and (10), we have

$$
\left[\begin{array}{l}
\bar{U}^{\prime} \\
\bar{T}^{\prime}
\end{array}\right]=\left[\begin{array}{ll}
M_{1} & M_{2} \\
M_{3} & M_{4}
\end{array}\right]\left[\begin{array}{l}
\bar{U} \\
\bar{T}
\end{array}\right]
$$

where $\bar{U}=\left[\begin{array}{ll}\bar{u}_{1} & \bar{u}_{2}\end{array}\right]^{T}, \bar{T}=\left[\begin{array}{ll}\bar{s}_{21} & \bar{s}_{22}\end{array}\right]^{T}$, the symbol "T" indicates the transpose of a matrix, the prime signifies differentiation with respect to $x_{2}$ and

$$
\begin{aligned}
& M_{1}=\left[\begin{array}{cc}
0 & -r_{2} \partial_{1} \\
-r_{1} \partial_{1} & 0
\end{array}\right], \quad M_{2}=\left[\begin{array}{cc}
\frac{1}{\bar{\gamma}_{2}} & 0 \\
0 & \frac{1}{\bar{\alpha}_{22}}
\end{array}\right], \\
& M_{3}=\left[\begin{array}{cc}
r_{3} \partial_{1}^{2}+\bar{\rho} \partial_{t}^{2} & 0 \\
0 & r_{4} \partial_{1}^{2}+\bar{\rho} \partial_{t}^{2}
\end{array}\right], \quad M_{4}=M_{1}^{T},
\end{aligned}
$$

here we introduce the natations $\partial_{1}=\partial / \partial x_{1}, \partial_{1}^{2}=\partial^{2} / \partial x_{1}^{2}, \partial_{t}^{2}=\partial^{2} / \partial x_{t}^{2}$.

$$
r_{1}=\frac{\bar{\alpha}_{12}}{\bar{\alpha}_{22}}, r_{2}=\frac{\bar{\gamma}_{*}}{\bar{\gamma}_{2}}, r_{3}=-\frac{\bar{\alpha}_{11} \bar{\alpha}_{22}-\bar{\alpha}_{12}^{2}}{\bar{\alpha}_{22}}, r_{4}=-\frac{\bar{\gamma}_{1} \bar{\gamma}_{2}-\bar{\gamma}_{*}^{2}}{\bar{\gamma}_{22}} \text {. }
$$

Eq. (11) is called the matrix equation for a compressible pre-stressed elastic layer in plane strain.

\subsection{Stroh formalism for a compressible pre-stressed elastic layer}

Now we consider the propagation of a plane wave traveling in the $x_{1}$-direction with velocity $c(>0)$ and wave number $k(>0)$. Then, the displacement components of the wave in the layer are sought in the form

$$
\bar{u}_{n}=\bar{U}_{n}(y) e^{i k\left(x_{1}-c t\right)}, \quad \bar{\sigma}_{n 2}=i k \bar{\Sigma}_{n}(y) e^{i k\left(x_{1}-c t\right)}, n=1,2, y=k x_{2} .
$$

Substituting (14) into (11) yields

$$
\bar{\xi}^{\prime}=i N \bar{\xi}, y \in[-\varepsilon, 0], \varepsilon=k h,
$$

where the prime signifies differentiation with respect to $y$ and

$$
\bar{\xi}=\left[\begin{array}{l}
\bar{U} \\
\bar{\Sigma}
\end{array}\right], \quad \bar{U}=\left[\begin{array}{l}
\bar{U}_{1} \\
\bar{U}_{2}
\end{array}\right], \quad \bar{\Sigma}=\left[\begin{array}{l}
\bar{\Sigma}_{1} \\
\bar{\Sigma}_{2}
\end{array}\right], \quad N=\left[\begin{array}{ll}
N_{1} & N_{2} \\
N_{3} & N_{4}
\end{array}\right],
$$

in which the matrices $N_{k}$ are given by

$$
N_{1}=\left[\begin{array}{cc}
0 & -r_{2} \\
-r_{1} & 0
\end{array}\right], N_{2}=\left[\begin{array}{cc}
\frac{1}{\bar{\gamma}_{2}} & 0 \\
0 & \frac{1}{\bar{\alpha}_{22}}
\end{array}\right], N_{3}=\left[\begin{array}{cc}
r_{3}+\bar{X} & 0 \\
0 & r_{4}+\bar{X}
\end{array}\right], N_{4}=N_{1}^{T},
$$

where $\bar{X}=\bar{\rho} c^{2}$. Eq. (15) is called Stroh's formalism [20] for an orthotropic elastic layer. From (15) it follows

$$
\bar{\xi}^{(n)}=i^{n} N^{n} \bar{\xi}, N^{n}:=\left[\begin{array}{ll}
N_{1}^{(n)} & N_{2}^{(n)} \\
N_{3}^{(n)} & N_{4}^{(n)}
\end{array}\right], y \in[-\varepsilon, 0], n=1,2,3, \ldots
$$


H/V ratio of Rayleigh waves in compressible pre-stressed elastic half-spaces coated with a thin layer: An approximate formula 67

\subsection{Relations between the traction and displacement amplitude vectors at two sides of the interface}

Let $\varepsilon:=k h$ be small (i.e., the layer is thin), then expanding into Taylor series $\bar{\xi}(-\varepsilon)$ at $y=0$ up to the third-order yields

$$
\bar{\xi}(-\varepsilon)=\bar{\xi}(0)-\varepsilon \bar{\xi}^{\prime}(0)+\frac{\varepsilon^{2}}{2} \bar{\xi}^{\prime \prime}(0)-\frac{\varepsilon^{3}}{6} \bar{\xi}^{\prime \prime \prime}(0)
$$

Substituting (18) into (19), we have

$$
\bar{\xi}(-\varepsilon)=\left[I-\varepsilon i N-\frac{\varepsilon^{2}}{2} N^{2}+\frac{\varepsilon^{3}}{6} i N^{3}\right] \bar{\xi}(0) .
$$

Eq. (20) can be written

$$
\begin{aligned}
& \bar{U}(-\varepsilon)=A \bar{U}(0)+B \bar{\Sigma}(0), \\
& \bar{\Sigma}(-\varepsilon)=C \bar{U}(0)+D \bar{\Sigma}(0),
\end{aligned}
$$

where

$$
\begin{aligned}
& A=\left[\begin{array}{cc}
1-\frac{\varepsilon^{2}}{2}\left(r_{6}+\bar{X} / \bar{\gamma}_{2}\right) & i\left(\varepsilon r_{2}-\frac{\varepsilon^{3}}{6}\left(t_{5}+t_{6} \bar{X}\right)\right) \\
i\left(\varepsilon r_{1}-\frac{\varepsilon^{3}}{6}\left(t_{1}+t_{2} \bar{X}\right)\right) & 1-\frac{\varepsilon^{2}}{2}\left(r_{8}+\bar{X} / \bar{\alpha}_{22}\right)
\end{array}\right], \\
& B=\left[\begin{array}{cc}
i\left[-\frac{1}{\bar{\gamma}_{2}}+\frac{\varepsilon^{3}}{6}\left(t_{9}+\bar{X} / \bar{\gamma}_{2}^{2}\right)\right] & \frac{\varepsilon^{2}}{2}\left(\frac{r_{1}}{\bar{\gamma}_{2}}+\frac{r_{2}}{\bar{\alpha}_{22}}\right) \\
\frac{\varepsilon^{2}}{2}\left(\frac{r_{1}}{\bar{\gamma}_{2}}+\frac{r_{2}}{\bar{\alpha}_{22}} c\right) & i\left[-\frac{\varepsilon}{\bar{\alpha}_{22}}+\frac{\varepsilon^{3}}{6}\left(t_{10}+\bar{X} / \bar{\alpha}_{22}^{2}\right)\right]
\end{array}\right], \\
& C=\left[\begin{array}{cc}
i\left[-\varepsilon\left(r_{3}+\bar{X}\right)+\frac{\varepsilon^{3}}{6}\left(t_{3}+t_{4} \bar{X}+\frac{\bar{X}^{2}}{\bar{\gamma}_{2}}\right)\right] & \frac{\varepsilon^{2}}{2}\left(r_{7}+r_{5} \bar{X}\right) \\
\frac{\varepsilon^{2}}{2}\left(r_{7}+r_{5} \bar{X}\right) & i\left[-\varepsilon\left(r_{4}+\bar{X}\right)+\frac{\varepsilon^{3}}{6}\left(t_{7}+t_{8} \bar{X}+\frac{\bar{X}^{2}}{\bar{\alpha}_{22}}\right)\right]
\end{array}\right], \\
& D=\left[\begin{array}{cc}
1-\frac{\varepsilon^{2}}{2}\left(r_{6}+\frac{\bar{X}}{\bar{\gamma}_{2}}\right) & i \varepsilon r_{1} \\
i \varepsilon r_{2} & 1-\frac{\varepsilon^{2}}{2}\left(r_{8}+\frac{\bar{X}}{\bar{\alpha}_{22}}\right)
\end{array}\right] \text {, }
\end{aligned}
$$

with

$$
\begin{aligned}
& r_{5}=r_{1}+r_{2}, \quad r_{6}=\frac{r_{3}}{\bar{\gamma}_{2}}+r_{1} r_{2}, \quad r_{7}=r_{2} r_{3}+r_{1} r_{4}, \quad r_{8}=\frac{r_{4}}{\bar{\alpha}_{22}}+r_{1} r_{2}, \\
& t_{3}=r_{1} r_{7}+r_{3} r_{6}, t_{4}=\frac{r_{3}}{\bar{\gamma}_{2}}+r_{1} r_{5}+r_{6}, t_{7}=r_{2} r_{7}+r_{4} r_{8}, t_{8}=\frac{r_{4}}{\bar{\alpha}_{22}}+r_{2} r_{5}+r_{8},
\end{aligned}
$$

At the surface $x_{2}=-h$ of the layer, the traction is free: $\bar{\Sigma}(-\varepsilon)=0$, so that (22) can written as

$$
C \bar{U}(0)+D \bar{\Sigma}(0)=0 .
$$

The (25) is the relation between the traction and displacement amplitude vectors at layerside of the interface $y=0$. 
According to Ogden and Vinh [5], the displacement components of Rayleigh waves in the half-space are given by

$$
u_{n}=U_{n}(y) e^{i k\left(x_{1}-c t\right)}, \quad \sigma_{n 2}=i k \Sigma_{n}(y) e^{i k\left(x_{1}-c t\right)}, n=1,2
$$

where

$$
\begin{gathered}
U_{1}(y)=B_{1} e^{-b_{1} y}+B_{2} e^{-b_{2} y}, \quad U_{2}(y)=i\left(\alpha_{1} B_{1} e^{-b_{1} y}+\alpha_{2} B_{2} e^{-b_{2} y}\right), \\
\Sigma_{1}(y)=i\left(\beta_{1} B_{1} e^{-b_{1} y}+\beta_{2} B_{2} e^{-b_{2} y}\right), \quad \Sigma_{2}(y)=\eta_{1} B_{1} e^{-b_{1} y}+\eta_{2} B_{2} e^{-b_{2} y},
\end{gathered}
$$

here $\alpha_{k}, \beta_{k}, \eta_{k}$ are defined by

$$
\begin{aligned}
& \alpha_{k}=\frac{\left(\alpha_{12}+\gamma_{*}\right) b_{k}}{\alpha_{22} b_{k}^{2}-\gamma_{1}+X}, \quad k=1,2, \quad X=\rho c^{2}, \\
& \beta_{k}=\gamma_{2} b_{k}+\gamma_{*} \alpha_{k}, \quad \eta_{k}=\alpha_{12}-\alpha_{22} \alpha_{k} b_{k},
\end{aligned}
$$

and $b_{1}, b_{2}$ are to with positive real parts roots of the equation

$$
\gamma_{2} \alpha_{22} b^{4}+\left\{\left(\alpha_{12}+\gamma_{*}\right)^{2}+\alpha_{22}\left(X-\alpha_{11}\right)+\gamma_{2}\left(X-\gamma_{1}\right)\right\} b^{2}+\left(X-\alpha_{11}\right)\left(X-\gamma_{1}\right)=0 .
$$

From (30), we obtain

$$
\begin{aligned}
& b_{1}^{2}+b_{2}^{2}=-\frac{\left(\alpha_{12}+\gamma_{*}\right)^{2}+\alpha_{22}\left(X-\alpha_{11}\right)+\gamma_{2}\left(X-\gamma_{1}\right)}{\alpha_{22} \gamma_{2}}:=S, \\
& b_{1}^{2} \cdot b_{2}^{2}=\frac{\left(X-\alpha_{11}\right)\left(X-\gamma_{1}\right)}{\alpha_{22} \gamma_{2}}:=P .
\end{aligned}
$$

It is not difficult to verify that if a Rayleigh wave exists $\left(b_{1}, b_{2}\right.$ having positive real parts) then

$$
0<X<\min \left\{\alpha_{11}, \gamma_{11}\right\}, P>0, S+2 \sqrt{P}>0, b_{1} \cdot b_{2}=\sqrt{P}, \quad b_{1}+b_{2}=\sqrt{S+2 \sqrt{P}} .
$$

Taking $x_{2}=0$ in (27) and (28) gives

$$
\begin{aligned}
& U_{1}(0)=B_{1}+B_{2}, \quad U_{2}(0)=i\left(\alpha_{1} B_{1}+\alpha_{2} B_{2}\right), \\
& \Sigma_{1}(0)=i\left(\beta_{1} B_{1}+\beta_{2} B_{2}\right), \quad \Sigma_{2}(0)=\eta_{1} B_{1}+\eta_{2} B_{2} .
\end{aligned}
$$

Eliminating $B_{1}, B_{2}$ from Eqs. (33) we have

$$
\Sigma(0)=H U(0), \quad H=\left[\begin{array}{cc}
i \frac{[\alpha ; \beta]}{[\alpha]} & \frac{[\beta]}{[\alpha]} \\
\frac{[\alpha ; \eta]}{[\alpha]} & -i \frac{[\eta]}{[\alpha]}
\end{array}\right],
$$

where

$$
\begin{array}{ll}
\frac{[\alpha, \beta]}{[\alpha]}=\frac{\gamma_{2}\left(\alpha_{11}-X\right)\left(b_{1}+b_{2}\right)}{\alpha_{11}-X+\gamma_{2} b_{1} b_{2}}, & \frac{[\eta]}{[\alpha]}=\frac{\gamma_{2} \alpha_{22} b_{1} b_{2}\left(b_{1}+b_{2}\right)}{\alpha_{11}-X+\gamma_{2} b_{1} b_{2}} \\
\frac{[\beta]}{[\alpha]}=\frac{\gamma_{*}\left(\alpha_{11}-X\right)-\gamma_{2} \alpha_{12} b_{1} b_{2}}{\alpha_{11}-X+\gamma_{2} b_{1} b_{2}}, & \frac{[\alpha, \eta]}{[\alpha]}=-\frac{[\beta]}{[\alpha]}
\end{array}
$$

Here, for the seeking of simplicity, we use the notations

$$
[f ; g]=f_{2} g_{1}-f_{1} g_{2}, \quad[\phi]=\phi_{2}-\phi_{1} .
$$


$H / V$ ratio of Rayleigh waves in compressible pre-stressed elastic half-spaces coated with a thin layer: An approximate formula 69

Eq. (34) is the relation between the traction and displacement amplitude vectors at the half-space-side of the interface $y=0$.

\section{AN APPROXIMATE FORMULAS FOR THE H/V RATIO}

The layer and half-space are taken to be bonded along the interface $y=0$, so that the displacement and traction are continuous across the interface. Thus,

$$
\bar{U}(0)=U(0), \quad \bar{\Sigma}(0)=\Sigma(0) .
$$

Substituting (34) into (21), (25) and taking into account (37) leads to

$$
\begin{array}{ll}
\bar{U}(-\varepsilon)=Q U(0), & Q=A+B H, \\
Z U(0)=0, & Z=C+D H,
\end{array}
$$

where

$$
\begin{aligned}
& Q_{11}=1+\varepsilon\left\{\frac{1}{\bar{\gamma}_{2}} \frac{[\alpha, \beta]}{[\alpha]}\right\}+\frac{\varepsilon^{2}}{2}\left\{r_{6}+\frac{\bar{X}}{\bar{\gamma}_{2}}-\left(\frac{r_{1}}{\bar{\gamma}_{2}}+\frac{r_{2}}{\bar{\alpha}_{22}}\right) \frac{[\beta]}{[\alpha]}\right\}-\frac{\varepsilon^{3}}{6}\left\{\left(t_{9}+\frac{\bar{X}}{\bar{\gamma}_{2}^{2}}\right) \frac{[\alpha, \beta]}{[\alpha]}\right\}, \\
& Q_{12}=i\left[\varepsilon\left\{r_{2}-\frac{1}{\bar{\gamma}_{2}} \frac{[\beta]}{[\alpha]}\right\}-\frac{\varepsilon^{2}}{2}\left\{\left(\frac{r_{1}}{\bar{\gamma}_{2}}+\frac{r_{2}}{\bar{\alpha}_{22}}\right) \frac{[\eta]}{[\alpha]}\right\}+\frac{\varepsilon^{3}}{6}\left\{-t_{5}-t_{6} \bar{X}+\left(t_{9}+\frac{\bar{X}}{\bar{\gamma}_{2}^{2}}\right) \frac{[\beta]}{[\alpha]}\right\}\right], \\
& Q_{21}=i\left[\varepsilon\left\{r_{1}+\frac{1}{\bar{\alpha}_{22}} \frac{[\beta]}{[\alpha]}\right\}+\frac{\varepsilon^{2}}{2}\left\{\left(\frac{r_{1}}{\bar{\gamma}_{2}}+\frac{r_{2}}{\bar{\alpha}_{22}}\right) \frac{[\alpha, \beta]}{[\alpha]}\right\}-\frac{\varepsilon^{3}}{6}\left\{t_{1}+t_{2} \bar{X}+\left(t_{10}+\frac{\bar{X}}{\bar{\alpha}_{22}^{2}}\right) \frac{[\beta]}{[\alpha]}\right\}\right], \\
& Q_{22}=1-\varepsilon\left\{\frac{1}{\bar{\alpha}_{22}} \frac{[\eta]}{[\alpha]}\right\}+\frac{\varepsilon^{2}}{2}\left\{-r_{8}-\frac{\bar{X}}{\bar{\alpha}_{22}}+\left(\frac{r_{1}}{\bar{\gamma}_{2}}+\frac{r_{2}}{\bar{\alpha}_{22}}\right) \frac{[\beta]}{[\alpha]}\right\}+\frac{\varepsilon^{3}}{6}\left\{\left(t_{10}+\frac{\bar{X}}{\bar{\alpha}_{22}^{2}}\right) \frac{[\eta]}{[\alpha]}\right\}
\end{aligned}
$$

and

$$
\begin{aligned}
& Z_{11}=i\left[\frac{[\alpha, \beta]}{[\alpha]}-\varepsilon\left\{r_{3}+\bar{X}+r_{1} \frac{[\beta]}{[\alpha]}\right\}-\frac{\varepsilon^{2}}{2}\left\{\left(r_{6}+\frac{\bar{X}}{\bar{\gamma}_{2}}\right) \frac{[\alpha, \beta]}{[\alpha]}\right\}+\frac{\varepsilon^{3}}{6}\left\{t_{3}+t_{4} \bar{X}+\frac{\bar{X}^{2}}{\bar{\gamma}_{2}}\right\}\right], \\
& Z_{12}=\frac{[\beta]}{[\alpha]}+\varepsilon\left\{r_{1} \frac{[\eta]}{[\alpha]}\right\}+\frac{\varepsilon^{2}}{2}\left\{r_{7}+r_{5} \bar{X}-\left(r_{6}+\frac{\bar{X}}{\bar{\gamma}_{2}}\right) \frac{[\beta]}{[\alpha]}\right\}, \\
& Z_{21}=-\frac{[\beta]}{[\alpha]}-\varepsilon\left\{r_{2} \frac{[\alpha, \beta]}{[\alpha]}\right\}+\frac{\varepsilon^{2}}{2}\left\{r_{7}+r_{5} \bar{X}+\left(r_{8}+\frac{\bar{X}}{\bar{\alpha}_{22}}\right) \frac{[\beta]}{[\alpha]}\right\}, \\
& Z_{22}=i\left[\frac{[\eta]}{[\alpha]}+\varepsilon\left\{-r_{4}-\bar{X}+r_{2} \frac{[\beta]}{[\alpha]}\right\}+\frac{\varepsilon^{2}}{2}\left\{\left(r_{8}+\frac{\bar{X}}{\bar{\gamma}_{2}}\right) \frac{[\eta]}{[\alpha]}\right\}+\frac{\varepsilon^{3}}{6}\left\{t_{7}+t_{8} \bar{X}+\frac{\bar{X}^{2}}{\bar{\alpha}_{22}}\right\}\right] .
\end{aligned}
$$

From (38) and (40), it follows

$$
\chi:=\left|\frac{\bar{u}_{1}(-h)}{\bar{u}_{2}(-h)}\right|=\left|\frac{\bar{U}_{1}(-\varepsilon)}{\bar{U}_{2}(-\varepsilon)}\right|=\left|\frac{Q_{12} Z_{11}-Q_{11} Z_{12}}{Q_{22} Z_{11}-Q_{21} Z_{12}}\right|,
$$

where $Z_{11}, Z_{12}$ are defined by (40), elements of matrix $Q$ are defined by (39). Note that, due to $|Z|=0$, therefore $\chi$ can be given by an alternative formula

$$
\chi:=\left|\frac{Q_{12} Z_{21}-Q_{11} Z_{22}}{Q_{22} Z_{21}-Q_{21} Z_{22}}\right| .
$$


After some manipulations, we arrive at the desired approximate formula of third order for the $\mathrm{H} / \mathrm{V}$ ratio, namely

where

$$
\chi=\left|\frac{A_{0}+A_{1} \varepsilon+A_{2} \frac{\varepsilon^{2}}{2}+A_{3} \frac{\varepsilon^{3}}{6}+O\left(\varepsilon^{4}\right)}{B_{0}+B_{1} \varepsilon+B_{2} \frac{\varepsilon^{2}}{2}+B_{3} \frac{\varepsilon^{3}}{6}+O\left(\varepsilon^{4}\right)}\right| .
$$

$$
\begin{aligned}
& A_{0}=-\frac{[\beta]}{[\alpha]} \\
& A_{1}=-r_{2} \frac{[\alpha, \beta]}{[\alpha]}-r_{1} \frac{[\eta]}{[\alpha]} \\
& A_{2}=2 r_{2} r_{3}-r_{7}+\left(2 r_{2}-r_{5}\right) \bar{X}+4 r_{1} r_{2} \frac{[\beta]}{[\alpha]}+\left(\frac{r_{2}}{\bar{\alpha}_{22}}-\frac{r_{1}}{\bar{\gamma}_{2}}\right) \frac{[\beta]^{2}+[\alpha, \beta][\eta]}{[\alpha]^{2}}, \\
& A_{3}=3\left[r_{1}^{2} r_{2}-\frac{r_{2}\left(r_{3}+\bar{X}\right)}{\bar{\alpha}_{22}}\right] \frac{[\eta]}{[\alpha]}+\left[t_{5}+3 r_{2} r_{6}+t_{6} \bar{X}+3 \frac{-r_{7}+\left(r_{2}-r_{5}\right) \bar{X}}{\bar{\gamma}_{2}}\right] \frac{[\alpha ; \beta]}{[\alpha]}, \\
& B_{0}=\frac{[\alpha, \beta]}{[\alpha]} \\
& B_{1}=-r_{3}-\bar{X}-2 r_{1} \frac{[\beta]}{[\alpha]}-\frac{1}{\bar{\alpha}_{22}} \frac{[\beta]^{2}+[\alpha, \beta][\eta]}{[\alpha]^{2}}, \\
& B_{2}=-\left[r_{6}+r_{8}+\bar{X}\left(\frac{1}{\bar{\alpha}_{22}}+\frac{1}{\bar{\gamma}_{2}}\right)\right] \frac{[\alpha, \beta]}{[\alpha]}+2\left(\frac{r_{3}+\bar{X}}{\bar{\alpha}_{22}}-r_{1}^{2}\right) \frac{[\eta]}{[\alpha]}, \\
& B_{3}=t_{3}+3\left(r_{3} r_{8}-r_{1} r_{7}\right)+\left(t_{4}+3 r_{8}-3 r_{1} r_{5}+\frac{3 r_{3}}{\bar{\alpha}_{22}}\right) \bar{X}+\left(\frac{3}{\bar{\alpha}_{22}}+\frac{1}{\bar{\gamma}_{2}}\right) \bar{X}^{2} \\
& +3\left(r_{1}\left[r_{6}+\frac{\bar{X}}{\bar{\gamma}_{2}}\right]-\frac{r_{7}+r_{5} \bar{X}}{\bar{\alpha}_{22}}+r_{1}\left[r_{8}+\frac{\bar{X}}{\bar{\alpha}_{22}}\right]-\left[\frac{r_{1}}{\bar{\gamma}_{2}}+\frac{r_{2}}{\bar{\alpha}_{22}}\right]\left(r_{3}+\bar{X}\right)+\frac{t_{1}+t_{2} \bar{X}}{3}\right) \frac{[\beta]}{[\alpha]} \\
& +\left(t_{10}-3 r_{1}\left[\frac{r_{1}}{\bar{\gamma}_{2}}+\frac{r_{2}}{\bar{\alpha}_{22}}\right]+\frac{3}{\bar{\alpha}_{22}}\left[r_{6}+\frac{\bar{X})}{\bar{\gamma}_{2}}\right]+\frac{\bar{X}}{\bar{\alpha}_{22}^{2}}\right) \frac{[\beta]^{2}+[\alpha, \beta][\eta]}{[\alpha]^{2}} \text {. }
\end{aligned}
$$

In the dimensionless form Eq. (43) is of the form

$$
\chi=\left|\frac{\bar{A}_{0}+\bar{A}_{1} \varepsilon+\bar{A}_{2} \frac{\varepsilon^{2}}{2}+\bar{A}_{3} \frac{\varepsilon^{3}}{6}+O\left(\varepsilon^{4}\right)}{\bar{B}_{0}+\bar{B}_{1} \varepsilon+\bar{B}_{2} \frac{\varepsilon^{2}}{2}+\bar{B}_{3} \frac{\varepsilon^{3}}{6}+O\left(\varepsilon^{4}\right)}\right| .
$$

in which the coefficients $\bar{A}_{k}, \bar{B}_{k}(k=0,1,2,3)$ are given by (63) in Appendix and they depend on the following dimensionless parameters

$$
\begin{aligned}
& e_{1}=\frac{\alpha_{11}}{\gamma_{1}}, e_{2}=\frac{\alpha_{22}}{\gamma_{1}}, e_{3}=\frac{\alpha_{12}}{\gamma_{1}}, \bar{e}_{1}=\frac{\bar{\alpha}_{11}}{\bar{\gamma}_{1}}, \bar{e}_{2}=\frac{\bar{\gamma}_{1}}{\bar{\alpha}_{22}}, \bar{e}_{3}=\frac{\bar{\alpha}_{12}}{\bar{\gamma}_{1}}, \\
& c_{2}=\sqrt{\frac{\gamma_{1}}{\rho}}, \bar{c}_{2}=\sqrt{\frac{\bar{\gamma}_{1}}{\bar{\rho}}}, r_{v}=\frac{c_{2}}{\bar{c}_{2}}, x=\frac{X}{c_{66}}, \bar{x}=\frac{\bar{X}}{\bar{\gamma}_{1}}, \bar{x}=r_{v}^{2} x .
\end{aligned}
$$


It is clear that the $\mathrm{H} / \mathrm{V}$ ratio $\chi$ depends on 9 dimensionless parameters: $e_{k}, \bar{e}_{k}(k=1,2,3)$, $r_{\mu}, r_{v}$ and $\varepsilon$ which are subjected the inequalities [21]

$$
e_{k}>0, \bar{e}_{k}>0(k=1,2), e_{1} e_{2}-e_{3}^{2}>0, \bar{e}_{1} / \bar{e}_{2}-\bar{e}_{3}^{2}>0
$$

Note that the $\mathrm{H} / \mathrm{V}$ ratio $\chi$ depends on the dimensionless Rayleigh wave velocity $x$ that is a solution of the secular equation (35) in [21] and it depend also on 9 dimensionless parameters mentioned above.

When $\varepsilon=0$, from (43) we have

$$
\chi=\left|\frac{\bar{A}_{0}}{\bar{B}_{0}}\right|=\left|\frac{[\beta]}{[\alpha ; \beta]}\right| .
$$

On the other hand, from (34) and $\Sigma(0)=0$ we have $|H|=0$, i.e.,

$$
[\beta]^{2}+[\alpha ; \beta][\eta]=0
$$

Using of (50) we have

$$
\chi^{2}=\frac{[\beta]^{2}}{[\alpha ; \beta]^{2}}=-\frac{[\eta]}{[\alpha ; \beta]}=\frac{\alpha_{22} b_{1} b_{2}}{\alpha_{11}-X}=\sqrt{\frac{\alpha_{22}}{\gamma_{2}}} \sqrt{\frac{\gamma_{1}-X}{\alpha_{11}-X}}
$$

When the half-spaces the half-space is isotropic, formula (51) is simplified to

$$
\chi=\sqrt[4]{\frac{1-x}{1-\gamma x}}, \gamma=\frac{\mu}{\lambda+2 \mu} .
$$

Eq. (52) is Eq. (12) in [15].

When the layer and the half-space are both unstressed isotropic

$$
\begin{aligned}
& \gamma_{1}=\gamma_{2}=\gamma_{*}=\mu, \alpha_{11}=\alpha_{22}=\lambda+2 \mu, \alpha_{12}=\lambda, \gamma=\frac{\mu}{\lambda+2 \mu}, \\
& \bar{\gamma}_{1}=\bar{\gamma}_{2}=\bar{\gamma}_{*}=\bar{\mu}, \bar{\alpha}_{11}=\bar{\alpha}_{22}=\bar{\lambda}+2 \bar{\mu}, \bar{\alpha}_{12}=\bar{\lambda}, \bar{\gamma}=\frac{\bar{\mu}}{\bar{\lambda}+2 \bar{\mu}} .
\end{aligned}
$$

With the help of (53) and (47) one can show that

$$
\begin{aligned}
& x=\frac{\rho c^{2}}{\mu}, e_{1}=e_{2}=\frac{1}{\gamma}, e_{3}=\frac{1}{\gamma}-2, e_{4}=e_{5}=1, c_{2}=\sqrt{\frac{\mu}{\rho}}, \bar{c}_{2}=\sqrt{\frac{\bar{\mu}}{\bar{\rho}}}, \\
& \bar{e}_{1}=\frac{1}{\bar{\gamma}}, \bar{e}_{2}=\bar{\gamma}, \bar{e}_{3}=\frac{1}{\bar{\gamma}}-2, \bar{e}_{4}=\bar{e}_{5}=1, r_{\mu}=\frac{\mu}{\bar{\mu}^{\prime}}, r_{v}=\frac{c_{2}}{\bar{c}_{2}}, \bar{x}=r_{v}^{2} x, \\
& S=2-(1+\gamma) x, \quad P=(1-\gamma x)(1-x),
\end{aligned}
$$


The H/V ratio is defined by Eq. (46) with $A_{i}$ and $B_{i}(i=0,1,2,3)$ are given by

$$
\begin{aligned}
\bar{A}_{0}= & -\frac{1}{r_{\mu}} \frac{1-\gamma x-(1-2 \gamma) \sqrt{P}}{1-\gamma x+\gamma \sqrt{P}}, \\
\bar{A}_{1}= & -\frac{1}{r_{\mu}}\left[\frac{(1-\gamma x) \sqrt{S+2 \sqrt{P}}}{1-\gamma x+\gamma \sqrt{P}}-(1-2 \bar{\gamma}) \frac{\sqrt{S+2 \sqrt{P}} \sqrt{P}}{1-\gamma x+\gamma \sqrt{P}}\right], \\
\bar{A}_{2}= & 4(\bar{\gamma}-1)+2 \bar{\gamma} r_{v}^{2} x-\frac{4(1-2 \bar{\gamma})}{r_{\mu}} \frac{1-\gamma x-(1-2 \gamma) \sqrt{P}}{1-\gamma x+\gamma \sqrt{P}} \\
& +\frac{3 \bar{\gamma}-1}{r_{\mu}^{2}} \frac{[1-\gamma x-(1-2 \gamma) \sqrt{P}]^{2}-(1-\gamma x)(S+2 \sqrt{P}) \sqrt{P}}{[1-\gamma x+\gamma \sqrt{P}]^{2}}, \\
\bar{A}_{3}= & \frac{1}{r_{\mu}}\left[3\left(\bar{\gamma} r_{v}^{2} x-1\right) \frac{\sqrt{S+2 \sqrt{P}} \sqrt{P}}{1-\gamma x+\gamma \sqrt{P}}+\left[(5 \bar{\gamma}-1) r_{v}^{2} x-4 \bar{\gamma}\right] \frac{(1-\gamma x) \sqrt{S+2 \sqrt{P}}}{1-\gamma x+\gamma \sqrt{P}}\right],
\end{aligned}
$$

and

$$
\begin{aligned}
\bar{B}_{0}= & \frac{1}{r_{\mu}} \frac{(1-\gamma x) \sqrt{S+2 \sqrt{P}}}{1-\gamma x+\gamma \sqrt{P}}, \\
\bar{B}_{1}= & -4(\bar{\gamma}-1)-r_{v}^{2} x-\frac{2(1-2 \bar{\gamma})}{r_{\mu}} \frac{1-\gamma x-(1-2 \gamma) \sqrt{P}}{1-\gamma x+\gamma \sqrt{P}} \\
& -\frac{\bar{\gamma}}{r_{\mu}^{2}} \frac{[1-\gamma x-(1-2 \gamma) \sqrt{P}]^{2}-(1-\gamma x)(S+2 \sqrt{P}) \sqrt{P}}{[1-\gamma x+\gamma \sqrt{P}]^{2}}, \\
\bar{B}_{2}= & \frac{1}{r_{\mu}}\left[\left[2-(1+\bar{\gamma}) r_{v}^{2} x\right] \frac{(1-\gamma x) \sqrt{S+2 \sqrt{P}}}{1-\gamma x+\gamma \sqrt{P}}+2\left(1-\bar{\gamma} r_{v}^{2} x\right) \frac{\sqrt{S+2 \sqrt{P}} \sqrt{P}}{1-\gamma x+\gamma \sqrt{P}}\right], \\
\bar{B}_{3}= & 8(\bar{\gamma}-1)+(1+3 \bar{\gamma}) r_{v}^{4} x^{2}+4\left(\bar{\gamma}^{2}-2\right) r_{v}^{2} x \\
& +\frac{\left(-2 \bar{\gamma}^{2}-6 \bar{\gamma}+1\right) r_{v}^{2} x+4 \bar{\gamma}+31-\gamma x-(1-2 \gamma) \sqrt{P}}{r_{\mu}} \\
& +\frac{-2 \bar{\gamma}-2+\left(\bar{\gamma}^{2}+3 \gamma\right) r_{v}^{2} x}{r_{\mu}^{2}} \frac{[1-\gamma x-(1-2 \gamma) \sqrt{P}]^{2}-(1-\gamma x)(S+2 \sqrt{P}) \sqrt{P}}{[1-\gamma x+\gamma \sqrt{P}]^{2}} .
\end{aligned}
$$

\section{NUMERICAL RESULTS}

We consider the case of isotropic pre-strains (equibiaxial deformation), where (see $[22,23])$

$$
\lambda_{1}=\lambda_{2}=\lambda, \quad \bar{\lambda}_{1}=\bar{\lambda}_{2}=\bar{\lambda} \text {. }
$$


From (3)-(6), we have

$$
\begin{aligned}
& \alpha_{11}=\alpha_{22}=\frac{\partial^{2} W}{\partial \lambda_{1}^{2}}, \\
& \gamma_{1}=\gamma_{2}=\frac{1}{2}\left(\frac{\partial^{2} W}{\partial \lambda_{1}^{2}}-\frac{\partial^{2} W}{\partial \lambda_{1} \partial \lambda_{2}}+\frac{1}{\lambda} \frac{\partial W}{\partial \lambda}\right), \\
& \gamma^{*}=\frac{1}{2}\left(\frac{\partial^{2} W}{\partial \lambda_{1}^{2}}-\frac{\partial^{2} W}{\partial \lambda_{1} \partial \lambda_{2}}-\frac{1}{\lambda} \frac{\partial W}{\partial \lambda_{1}}\right),
\end{aligned}
$$

for the half-space and there are similar expressions for the layer. The continuity of the normal stress (see [22]) implies that

$$
\bar{\lambda} \bar{\lambda}_{3} \frac{\partial W}{\partial \lambda_{2}}=\lambda \lambda_{3} \frac{\partial \bar{W}}{\partial \lambda_{2}}
$$

\subsection{A compressible neo-Hookean material}

We consider the strain-energy function given by (see [23])

$$
W=\frac{1}{2}\left(\lambda_{1}^{-2}+\lambda_{2}^{-2}+\lambda_{3}^{-2}-3-2 \ln \left(\lambda_{1} \lambda_{2} \lambda_{3}\right)\right)
$$

for the half-space and similarly for the layer.

From (56)-(58) and taking into account (47), we have

$$
\begin{aligned}
& e_{1}=e_{2}=\frac{\lambda^{2}+1}{\lambda^{2}}, e_{3}=\bar{e}_{3}=0, \quad e_{4}=\frac{1}{\lambda^{2}}, \quad e_{5}=\bar{e}_{5}=1, \bar{e}_{1}=\frac{\bar{\lambda}^{2}+1}{\bar{\lambda}^{2}}, \\
& \bar{e}_{2}=\frac{1}{\bar{e}_{1}}, \quad \bar{e}_{4}=\frac{1}{\bar{\lambda}^{2}}, \bar{\lambda}^{2}=\frac{\lambda^{2}}{\lambda^{2}+r\left(1-\lambda^{2}\right)} r_{\mu}=\frac{1}{r}, r_{v}=\sqrt{R\left(\lambda^{2}+r\left(1-\lambda^{2}\right)\right)} .
\end{aligned}
$$

\subsection{A Blatz-Ko material}

We now consider the strain-energy function for the half-space given by (see [23])

$$
W=\frac{1}{2}\left(\lambda_{1}^{-2}+\lambda_{2}^{-2}+\lambda_{3}^{-2}+2 \lambda_{1} \lambda_{2} \lambda_{3}-5\right),
$$

and similarly for the layer.

From (56)-(58) and taking into account (47), we have

$$
\begin{aligned}
& e_{1}=e_{2}=\bar{e}_{1}=3, e_{3}=\lambda^{4}, e_{4}=2-\lambda^{4}, e_{5}=1,{ }^{2} \bar{e}_{2}=1 / \bar{e}_{1}, \bar{e}_{4}=2-\bar{\lambda}^{4}, \bar{e}_{5}=1, \\
& \bar{\lambda}^{4}=\frac{\lambda^{4}}{\lambda^{4}+r\left(1-\lambda^{4}\right)}, r_{v}^{2}=\frac{R}{\sqrt{\lambda^{4}+r\left(1-\lambda^{4}\right)}}, r=\frac{\mu}{\bar{\mu}}, R=\frac{\mu \bar{\rho} \bar{\lambda}^{2}}{\bar{\mu} \rho \lambda^{2}} .
\end{aligned}
$$

Figs. 1, 2 present the dependence on $\varepsilon \in[0,1]$ of the $H / V$ ratio that is calculated by the exact formulas and by the third-order approximate formula (46) for the neo-Hookean strain-energy function and the Blatz-Ko strain-energy function. It is shown from the Fig. 1 and Fig. 2 that the obtained third-order approximate formula for the H/V ratio is a good approximation. 


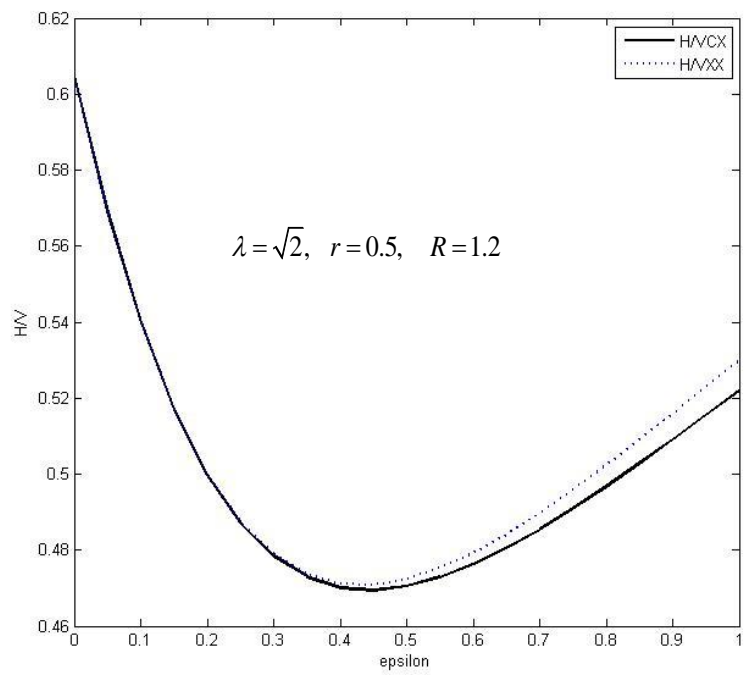

Fig. 1. Plots of $\chi$ calculated by the approximate secular equation of third-order (dashed line) (46), and by the exact secular equation for the neo-Hookean strain-energy function

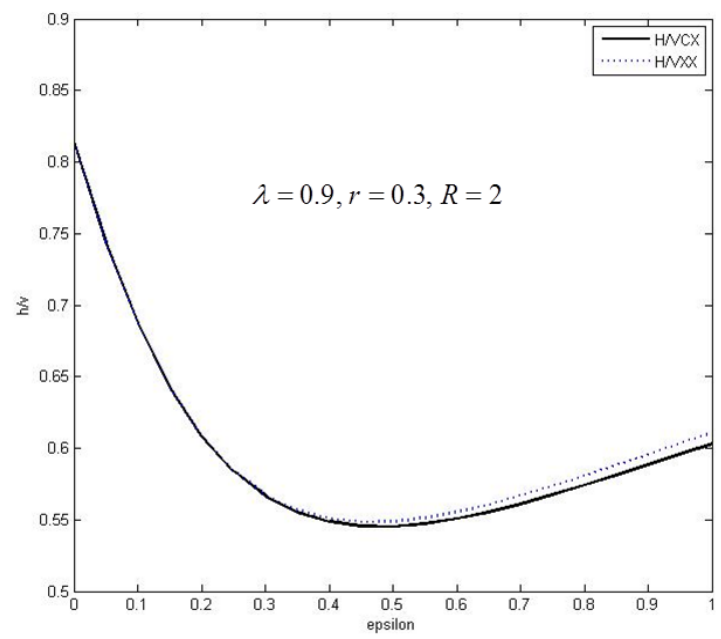

(a)

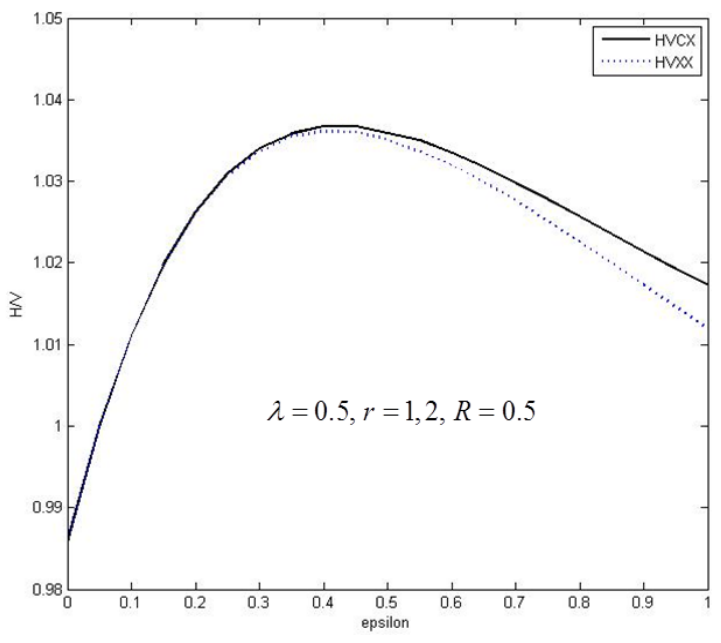

(b)

Fig. 2. Plots of $\chi$ calculated by the approximate secular equation of third-order (dashed line) (46), and by the exact secular equation

\section{CONCLUSIONS}

In this paper the propagation of Rayleigh waves in a pre-stressed elastic half-space coated by a thin pre-stressed elastic layer is investigated. An approximate formula for the $\mathrm{H} / \mathrm{V}$ ratio of third-order in terms of dimensionless thickness of the layer has been established by using the relations between the traction and displacement amplitude vectors of 
Rayleigh waves at two sides of the welded interface between the layer and the half-space. It is shown numerically that the obtained approximate formula is a good approximation. It will be employed as theoretical base for extracting the mechanical properties of thin films from measured values of the $\mathrm{H} / \mathrm{V}$ ratio.

\section{ACKNOWLEDGMENTS}

This research is funded by Vietnam National Foundation for Science and Technology Development (NAFOSTED) under grant number 107.02-2015.09.

\section{REFERENCES}

[1] M. Junge, J. Qu, and L. J. Jacobs. Relationship between Rayleigh wave polarization and state of stress. Ultrasonics, 44, (3), (2006), pp. 233-237. doi:10.1016/j.ultras.2006.03.004.

[2] P. C. Vinh and R. W. Ogden. On formulas for the Rayleigh wave speed. Wave Motion, 39, (3), (2004), pp. 191-197. doi:10.1016/j.wavemoti.2003.08.004.

[3] M. A. Dowaikh and R. W. Ogden. On surface waves and deformations in a compressible elastic half-space. Stability and Applied Analysis of Continuous Media, 1, (1), (1991), pp. 27-45.

[4] R. W. Ogden. Non-linear elastic deformations. Ellis Horwood, Chichester, (1984).

[5] P. C. Vinh and R. W. Ogden. Formulas for the Rayleigh wave speed in orthotropic elastic solids. Archives of Mechanics, 56, (3), (2004), pp. 247-265.

[6] R. W. Ogden and P. C. Vinh. On Rayleigh waves in incompressible orthotropic elastic solids. The Journal of the Acoustical Society of America, 115, (2), (2004), pp. 530-533. doi:10.1121/1.1636464.

[7] P. C. Vinh and R. W. Ogden. On the Rayleigh wave speed in orthotropic elastic solids. Meccanica, 40, (2), (2005), pp. 147-161. doi:10.1007/s11012-005-1603-6.

[8] P. C. Vinh and P. G. Malischewsky. An improved approximation of Bergmann's form for the Rayleigh wave velocity. Ultrasonics, 47, (1), (2007), pp. 49-54. doi:10.1016/j.ultras.2007.07.002.

[9] P. C. Vinh and P. G. Malischewsky. An approach for obtaining approximate formulas for the Rayleigh wave velocity. Wave Motion, 44, (7), (2007), pp. 549-562. doi:10.1016/j.wavemoti.2007.02.001.

[10] P. C. Vinh and P. G. Malischewsky. Improved approximations of the Rayleigh wave velocity. Journal of Thermoplastic Composite Materials, 21, (4), (2008), pp. 337-352. doi:10.1177/0892705708089479.

[11] P. C. Vinh. On formulas for the velocity of Rayleigh waves in prestrained incompressible elastic solids. Journal of Applied Mechanics, 77, (2), (2010). doi:10.1115/1.3197139.

[12] P. C. Vinh and P. T. H. Giang. On formulas for the Rayleigh wave velocity in pre-strained elastic materials subject to an isotropic internal constraint. International Journal of Engineering Science, 48, (3), (2010), pp. 275-289. doi:10.1016/j.ijengsci.2009.09.010.

[13] P. C. Vinh. On formulas for the Rayleigh wave velocity in pre-stressed compressible solids. Wave Motion, 48, (7), (2011), pp. 614-625. doi:10.1016/j.wavemoti.2011.04.015.

[14] P. C. Vinh and P. G. Malischewsky. Improved approximations for the rayleigh wave velocity in [-1, 0.5]. Vietnam Journal of Mechanics, 30, (4), (2008), pp. 347-358.

[15] P. G. Malischewsky and F. Scherbaum. Love's formula and H/V-ratio (ellipticity) of Rayleigh waves. Wave motion, 40, (1), (2004), pp. 57-67. doi:10.1016/j.wavemoti.2003.12.015.

[16] A. E. H. Love. Some problems of geodynamics. Cambridge University Press, (1911).

[17] P. G. Malischewsky, F. Wuttke, and A. Ziegert. The use of surface acoustic waves for nondestructive testing. Schriftenreihe Werkstoffwissenschaffen, 17, (2002), pp. 135-140. (in German). 
[18] L. M. Munirova and T. B. Yanovskaya. Spectral ratio of the horizontal and vertical Rayleigh wave components and its application to some problems of seismology. Izvestiia Physics of the Solid Earth, 37, (9), (2001), pp. 709-716.

[19] F. Scherbaum, K. G. Hinzen, and M. Ohrnberger. Determination of shallow shear wave velocity profiles in the Cologne, Germany area using ambient vibrations. Geophysical Journal International, 152, (3), (2003), pp. 597-612. doi:10.1046/j.1365-246x.2003.01856.x.

[20] A. N. Stroh. Steady state problems in anisotropic elasticity. Studies in Applied Mathematics, 41, (1-4), (1962), pp. 77-103. doi:10.1002/sapm196241177.

[21] P. C. Vinh and N. T. K. Linh. An approximate secular equation of generalized Rayleigh waves in pre-stressed compressible elastic solids. International Journal of Non-Linear Mechanics, 50, (2013), pp. 91-96. doi:10.1016/j.ijnonlinmec.2012.11.004.

[22] R. W. Ogden and D. A. Sotiropoulos. The effect of pre-stress on guided ultrasonic waves between a surface layer and a half-space. Ultrasonics, 34, (2-5), (1996), pp. 491-494. doi:10.1016/0041-624x(95)00102-9.

[23] D. G. Roxburgh and R. W. Ogden. Stability and vibration of pre-stressed compressible elastic plates. International Journal of Engineering Science, 32, (3), (1994), pp. 427-454. doi:10.1016/0020-7225(94)90133-3. 
H/V ratio of Rayleigh waves in compressible pre-stressed elastic half-spaces coated with a thin layer: An approximate formula 77

\section{APPENDIX}

The expressions of $\bar{A}_{k}, \bar{B}_{k}(k=0,1,2,3)$

$$
\begin{aligned}
& \bar{A}_{0}=\frac{1}{r_{\mu}} \frac{e_{5} e_{3} \sqrt{P}-e_{4}\left(e_{1}-x\right)}{e_{1}-x+e_{5} \sqrt{P}}, \\
& \bar{A}_{1}=\frac{1}{r_{\mu}} \frac{e_{5} \sqrt{S+2 \sqrt{P}}\left[r_{1} e_{2} \sqrt{P}-r_{2}\left(e_{1}-x\right)\right]}{e_{1}-x+e_{5} \sqrt{P}}, \\
& \bar{A}_{2}=2 r_{2} \bar{r}_{3}-\bar{r}_{7}+\left(2 r_{2}-r_{5}\right) r_{v}^{2} x+\frac{4 r_{1} r_{2}}{r_{\mu}} \frac{\left(e_{4}\left(e_{1}-x\right)-e_{3} e_{5} \sqrt{P}\right.}{e_{1}-x+e_{5} \sqrt{P}} \\
& +\frac{r_{2} \bar{e}_{2}-r_{1} \bar{e}_{5}}{r_{\mu}^{2}} \frac{\left[e_{4}\left(e_{1}-x\right)-e_{3} e_{5} \sqrt{P}\right]^{2}-e_{2} e_{5}^{2}\left(e_{1}-x\right) \sqrt{P}(S+2 \sqrt{P})}{\left[e_{1}-x+e_{5} \sqrt{P}\right]^{2}}, \\
& \bar{A}_{3}=\frac{3\left(r_{2} \bar{e}_{2}\left[\overline{r_{3}}+r_{v}^{2} x\right]-r_{1}^{2} r_{2}\right)}{r_{\mu}} \frac{e_{2} e_{5} \sqrt{P} \sqrt{S+2 \sqrt{P}}}{e_{1}-x+e_{5} \sqrt{P}} \\
& +\frac{t_{5}+3 r_{2} r_{6}-3 \bar{e}_{5} \bar{r}_{7}+\left(\bar{t}_{6}+3 r_{2} \bar{e}_{5}-3 \bar{e}_{5} r_{5}\right) r_{v}^{2} x}{r_{\mu}} \frac{e_{5}\left(e_{1}-x\right) \sqrt{S+2 \sqrt{P}}}{e_{1}-x+e_{5} \sqrt{P}}, \\
& \bar{B}_{0}=\frac{1}{r_{\mu}} \frac{e_{5}\left(e_{1}-x\right) \sqrt{S+2 \sqrt{P}}}{e_{1}-x+e_{5} \sqrt{P}}, \\
& \bar{B}_{1}=-\bar{r}_{3}-r_{v}^{2} x-\frac{2 r_{1}}{r_{\mu}} \frac{\left(e_{4}\left(e_{1}-x\right)-e_{3} e_{5} \sqrt{P}\right.}{e_{1}-x+e_{5} \sqrt{P}} \\
& -\frac{\bar{e}_{2}}{r_{\mu}^{2}} \frac{\left[e_{4}\left(e_{1}-x\right)-e_{3} e_{5} \sqrt{P}\right]^{2}-e_{2} e_{5}^{2}\left(e_{1}-x\right) \sqrt{P}(S+2 \sqrt{P})}{\left[e_{1}-x+e_{5} \sqrt{P}\right]^{2}}, \\
& \bar{B}_{2}=-\frac{r_{8}+r_{6}+\left(\bar{e}_{2}+\bar{e}_{5}\right) r_{v}^{2} x}{r_{\mu}} \frac{e_{5}\left(e_{1}-x\right) \sqrt{S+2 \sqrt{P}}}{e_{1}-x+e_{5} \sqrt{P}} \\
& +\frac{-2 \bar{e}_{2}\left(\bar{r}_{3}+r_{v}^{2} x\right)+2 r_{1}^{2}}{r_{\mu}} \frac{e_{2} e_{5} \sqrt{P} \sqrt{S+2 \sqrt{P}}}{e_{1}-x+e_{5} \sqrt{P}}, \\
& \bar{B}_{3}=\bar{t}_{3}+3 r_{8} \bar{r}_{3}-3 r_{1} \bar{r}_{7}+\left(t_{4}+3 r_{8}+3 \bar{r}_{3} \bar{e}_{2}-3 r_{1} r_{5}\right) r_{v}^{2} x+\left(\bar{e}_{5}+3 \bar{e}_{2}\right) r_{v}^{4} x^{2} \\
& +\left(\frac{3\left(r_{1} r_{6}-\bar{r}_{7} \bar{e}_{2}+r_{1} r_{8}-r_{1} \bar{r}_{3} \bar{e}_{5}-r_{2} \bar{r}_{3} \bar{e}_{2}\right)+t_{1}}{r_{\mu}}\right. \\
& \left.+\frac{\left[3\left(r_{1} \bar{e}_{5}-r_{5} \bar{e}_{2}+r_{1} \bar{e}_{2}-r_{1} \bar{e}_{5}-r_{2} \bar{e}_{2}\right)+\bar{t}_{2}\right] r_{v}^{2} x}{r_{\mu}}\right) \cdot \frac{e_{4}\left(e_{1}-x\right)-e_{3} e_{5} \sqrt{P}}{e_{1}-x+e_{5} \sqrt{P}}, \\
& +\frac{\bar{t}_{10}+3 \bar{e}_{2} r_{6}-3 r_{1}\left(r_{1} \bar{e}_{5}+r_{2} \bar{e}_{2}\right)+\left(\bar{e}_{2}^{2}+3 \bar{e}_{2} \bar{e}_{5}\right) r_{v}^{2} x}{r_{\mu}^{2}} \\
& \frac{\left[e_{4}\left(e_{1}-x\right)-e_{3} e_{5} \sqrt{P}\right]^{2}-e_{2} e_{5}^{2}\left(e_{1}-x\right) \sqrt{P}(S+2 \sqrt{P})}{\left[e_{1}-x+e_{5} \sqrt{P}\right]^{2}},
\end{aligned}
$$


and

$$
\begin{aligned}
& r_{1}=\bar{e}_{2} \bar{e}_{3}, r_{2}=\bar{e}_{4} \bar{e}_{5}, r_{3}=\left(\bar{e}_{2} \bar{e}_{3}^{2}-\bar{e}_{1}\right) \bar{\gamma}_{1}=\bar{r}_{3} \bar{\gamma}_{1}, r_{4}=\left(\bar{e}_{4}^{2} \bar{e}_{5}-1\right) \bar{\gamma}_{1}=\bar{r}_{4} \bar{\gamma}_{1}, \\
& r_{5}=\bar{e}_{2} \bar{e}_{3}+\bar{e}_{4} \bar{e}_{5}, r_{6}=\left(\bar{e}_{2} \bar{e}_{3}^{2}-\bar{e}_{1}\right) \bar{e}_{5}+\bar{e}_{2} \bar{e}_{3} \bar{e}_{4} \bar{e}_{5}, \\
& r_{7}=\left[\bar{e}_{4} \bar{e}_{5}\left(\bar{e}_{2} \bar{e}_{3}^{2}-\bar{e}_{1}\right)+\bar{e}_{1} \bar{e}_{2}\left(\bar{e}_{4}^{2} \bar{e}_{5}-1\right)\right] \bar{\gamma}_{1}=\bar{r}_{7} \bar{\gamma}_{1}, r_{8}=\left(\bar{e}_{4}^{2} \bar{e}_{5}-1\right) \bar{e}_{2}+\bar{e}_{2} \bar{e}_{3} \bar{e}_{4} \bar{e}_{5}, \\
& t_{1}=\bar{e}_{2} \bar{r}_{7}+r_{1} r_{6}, t_{2}=\frac{1}{\bar{\gamma}_{1}}\left(r_{5} \bar{e}_{2}+r_{1} \bar{e}_{5}\right)=\frac{\bar{t}_{2}}{\bar{\gamma}_{1}}, t_{3}=\left(r_{1} \bar{r}_{7}+\bar{r}_{3} r_{6}\right) \bar{\gamma}_{1}=\bar{t}_{3} \bar{\gamma}_{1}, \\
& t_{4}=\bar{r}_{3} \bar{e}_{5}+r_{1} r_{5}+r_{6}, t_{5}=\bar{r}_{7} \bar{e}_{5}+r_{2} r_{8}, t_{6}=\frac{1}{\bar{\gamma}_{1}}\left(r_{5} \bar{e}_{5}+r_{2} \bar{e}_{2}\right)=\frac{\bar{t}_{6}}{\bar{\gamma}_{1}}, \\
& t_{7}=\left(r_{2} \bar{r}_{7}+\bar{r}_{4} r_{8}\right) \bar{\gamma}_{1}=\bar{t}_{7} \bar{\gamma}_{1}, t_{8}=\bar{r}_{4} \bar{e}_{2}+r_{2} r_{5}+r_{8}, \\
& t_{9}=\frac{1}{\bar{\gamma}_{1}}\left(\left[r_{1} r_{2}+r_{6}\right] \bar{e}_{5}+r_{2}^{2} \bar{e}_{2}\right)=\frac{\bar{t}_{9}}{\bar{\gamma}_{1}}, \quad t_{10}=\frac{1}{\bar{\gamma}_{1}}\left(r_{1}^{2} \bar{e}_{5}+\left[r_{1} r_{2}+r_{8}\right] \bar{e}_{2}\right)=\frac{\bar{t}_{10}}{\bar{\gamma}_{1}}, \\
& S=\frac{e_{2}\left(e_{1}-x\right)+e_{5}(1-x)-\left(e_{3}+e_{4}\right)^{2}}{e_{2} e_{5}}, \quad P=\frac{\left(e_{1}-x\right)(1-x)}{e_{2} e_{5}}, \\
& b_{1} b_{2}=\sqrt{P}, b_{1}+b_{2}=\sqrt{S+2 \sqrt{P}}, \quad \frac{[\alpha, \beta]}{[\alpha]}=\gamma_{1} \frac{e_{5}\left(e_{1}-x\right) \sqrt{S+2 \sqrt{P}}}{e_{1}-x-\sqrt{P}}, \\
& \frac{[\beta]}{[\alpha]}=-\gamma_{1} \frac{e_{1}-x-e_{3} \sqrt{P}}{x-e_{1}-\sqrt{P}}, \quad \frac{[\eta]}{[\alpha]}=\gamma_{1} \frac{e_{2} \sqrt{P} \sqrt{S+2 \sqrt{P}}}{x-e_{1}-\sqrt{P}} \text {. }
\end{aligned}
$$

